\title{
Microwave Corona Breakdown Prediction in Arbitrarily-Shaped Waveguide Based Filters
}

\author{
T. Pinheiro-Ortega, J. Monge, S. Marini, J. Sanz, E. Sorolla, M. Mattes, Member, IEEE, \\ C. Vicente, Member, IEEE, J. Gil, Member, IEEE, V. E. Boria, Senior Member, IEEE, and B. Gimeno, Member, IEEE
}

\begin{abstract}
This letter describes an efficient algorithm to predict the RF gas breakdown power threshold in microwave devices with complex geometries. The two necessary calculations when investigating such a phenomenon have been performed: on the one hand, the computation of the electromagnetic fields inside the structure and, on the other hand, the determination of the breakdown onset itself. The electromagnetic fields are solved by means of modal techniques and coupled to the free electron continuity equation, which is solved by the Finite Element Method. Proceeding in this way, a very simple criterion to find out whether microwave corona breakdown will take place is derived. This numerical implementation of the developed algorithms has been tested with other theoretical approaches and with experimental measurements, showing very good agreement.
\end{abstract}

Index Terms-Electromagnetic simulation, gas discharge, high power, microwave breakdown threshold, microwave filters.

\section{INTRODUCTION}

$\mathbf{I}$ $\mathrm{N}$ satellites, problems regarding RF breakdown due to gas discharges [1] mainly arise during their launching due to the atmospheric conditions. In terms of hardware design, the interesting parameter to determine is the breakdown power threshold of the discharge in order to avoid it. In the last years, a big effort has been devoted to understand, model, and predict this breakdown onset (see e.g., [2]). In [3], a full numerical approach based on finite differences has been developed in order to analyze the gas breakdown onset in components based on rectangular waveguides. In this letter, we extend such a work in order to deal with more complex passive waveguide components based on arbitrary cross section shapes. By arbitrary shape it is understood any cross section different from rectangular or cir-

Manuscript received October 04, 2009; revised December 30, 2009. First published March 08, 2010; current version published April 07, 2010. This work was supported by the Ministerio de Educacion y Ciencia of Spain under Grant TEC2007-67630-C03-01/TCM and the "Programa Torres Quevedo" (PTQ08-03-08254).

T. Pinheiro-Ortega, C. Vicente, and J. Gil are with Aurora Software and Testing S.L., Valencia, Spain (e-mail: teresa.pinheiro@ aurorasat.es).

S. Marini is with the Departamento de Física, Ingeniería de Sistemas y Teoría de la Señal, Universidad de Alicante, Spain (e-mail: smarini@ua.es).

E. Sorolla and M. Mattes are with the Laboratoire d'Electromagnétisme et d'Acoustique, Ecole Polytechnique Fédérale de Lausanne, Switzerland (e-mail: michael.mattes@epfl.ch).

J. Monge, J. Sanz and V. E. Boria are with the Departamento de Comunicaciones-iTEAM, Universidad Politécnica de Valencia, Spain (e-mail: vboria@dcom.upv.es).

B. Gimeno is with Departamento de Física Aplicada-ICMUV, Universidad de Valencia, Spain (e-mail: benito.gimeno@uv.es).

Color versions of one or more of the figures in this letter are available online at http://ieeexplore.ieee.org.

Digital Object Identifier 10.1109/LMWC.2010.2042555 cular (i.e., ridged and multi-ridged waveguides are included in this definition). In order to carry out the gas discharge analysis, first of all, the electric field density inside the microwave device has to be computed. Afterwards, this field distribution is coupled to the electron density continuity equation, which is solved by the Finite Element Method (FEM) in order to determine the gas discharge RF power threshold.

\section{Computation of Electromagnetic Field Distribution}

For an accurate gas discharge analysis, it is indispensable to precisely know the electromagnetic field distribution inside the microwave device. The electromagnetic characterization of the component has been done, in this work, by means of a fullwave analysis approach, which ensures a high accuracy and efficiency, also for complex geometries like microwave filters. Such a method is based on the integral equation technique and microwave network theory outlined in [4].

In order to compute the electromagnetic fields inside hollow arbitrary waveguides, the BI-RME (Boundary Integral-Resonant Mode Expansion) method [4] has been used. This modal technique efficiently characterizes such arbitrarily-shaped waveguides from the solution of integral equations resulting into eigenvalue problems for both TM and TE modes. This method has been successfully applied to the full-wave modal analysis of any arbitrarily shaped waveguide in [5], and omitted here for brevity.

\section{GAS BREAKDOWN THEORY AND NUMERICAL ANALYSIS}

In order to calculate the gas discharge breakdown power threshold, one has to compute the free electron density originated by ionization of the gas molecules inside the waveguide structure. To do this, the continuity equation, which governs the evolution of the electron density, must be solved [1]

$$
\nabla^{2}(D n(\mathbf{r}, t))+\left(\nu_{i}-\nu_{a}\right) n(\mathbf{r}, t)+P=\frac{\partial n(\mathbf{r}, t)}{\partial t}
$$

where $n(\mathbf{r}, t)$ is the electron current density, $D$ is the diffusion coefficient, $\nu_{i}$ and $\nu_{a}$ stand for the ionization and attachment rates, respectively, and $P$ is the electron production rate from external sources.

Generally, the relevant parameters $\nu_{i}, \nu_{a}$ and $D$ depend on the microwave electric field $\left(E_{\mathrm{rms}}\right)$, the gas pressure $(p)$ and the frequency $(f=\omega /(2 \pi))$. However, in this work, we have assumed that the diffusion coefficient only depends on the pressure [2], and we have used the experimental coefficients for dry air published in [6]. These parameters are given parametrically in such a publication, which allows including them easily in any numerical algorithm. Equation (1) has to be solved using 
numerical methods if the particular device exhibits a complex geometry, and therefore the concomitant electric field is highly inhomogeneous. In this work, a finite element scheme has been used for solving (1) due to its feasibility for studying arbitrary geometries.

We assume that the initial and boundary conditions for the electron density are respectively given by

$$
\begin{aligned}
n(\mathbf{r}, t=0) & =n_{0} \quad \forall \mathbf{r} \in V, \\
n(\mathbf{r} \in \partial V, t) & =0 \quad \forall t
\end{aligned}
$$

where the volume $V$ represents the spatial domain of the problem, $\partial V$ being its surface.

As a first step, we apply the unilateral Laplace transform to (1). This way, the equation adopts the simplified expression

$$
s \hat{n}(\mathbf{r}, s)-n_{0}-D \nabla^{2}(\hat{n}(\mathbf{r}, s))-\Delta \nu(\mathbf{r}) \hat{n}(\mathbf{r}, s)-\frac{P}{s}=0
$$

where $\Delta \nu(\mathbf{r}) \equiv \nu_{i}(E(\omega, \mathbf{r}), p)-\nu_{a}(E(\omega, \mathbf{r}), p)$ and the transformed boundary condition is $\hat{n}(\mathbf{r} \in \partial V, s)=0 \quad \forall s$.

Secondly, we consider the volume $V$ and apply Garlekins method to (4). Then, we use Gauss theorem, imposing the boundary conditions at $\partial V$. By following this procedure, we obtain

$$
\begin{aligned}
& \int_{V} w s \hat{n}(\mathbf{r}, s) d V-\int_{V} w n_{0} d V \\
& \quad+\int_{V} D \nabla w \cdot \nabla \hat{n}(\mathbf{r}, s) d V \\
& \quad-\int_{V} w \Delta \nu(\mathbf{r}) \hat{n}(\mathbf{r}, s) d V-\int_{V} w \frac{P}{s} d V=0
\end{aligned}
$$

being $w$ the test function.

The discretization of (5) gives

$$
(s[M]+[K])\{\hat{N}(s)\}^{T}=\left\{N_{0}\right\}^{T}+\frac{1}{s}\{P\}^{T}
$$

where $[M]$ and $[K]$ are $L \times L$ matrices, whose elements are defined as

$$
\begin{aligned}
{[M]_{i j} } & =\int_{V}\{T\}_{i}\{T\}_{j} d V, \\
{[K]_{i j} } & =[R]_{i j}-[U]_{i j}, \\
{[R]_{i j} } & =D \int_{V}\{\nabla T\}_{i}\{\nabla T\}_{j} d V, \\
{[U]_{i j} } & =\int_{V}\{T\}_{i} \Delta \nu(\mathbf{r})\{T\}_{j} d V
\end{aligned}
$$

being $\{T\}_{i}$ a basis function, with $i, j=1, \ldots, L$. Also $\left\{N_{0}\right\}$ and $\{P\}$ are vectors whose elements are defined as

$$
\begin{aligned}
\left\{N_{0}\right\}_{i} & =\int_{V}\{T\}_{i} n_{0} d V, \\
\{P\}_{i} & =\int_{V}\{T\}_{i} P d V
\end{aligned}
$$

and the superscript $T$ denotes the transpose.

It is important to notice that the matrix $[K]$ involves the dynamics of the problem. It contains both a term related with the diffusion of electrons in the gas, and also a balancing term concerning the gain and loss of electrons by ionization and attachment respectively. The increase of the applied electric field inside the microwave devices, due to an enhancement on the input power, will affect the matrix $[U]$ and accordingly the matrix $[K]$.

The algebraic problem represented by (6) can be transformed in the following way:

$$
\begin{aligned}
\{\hat{N}(s)\}^{T}= & (s[M]+[K])^{-1}\left(\left\{N_{0}\right\}^{T}+\frac{1}{s}\{P\}^{T}\right) \\
= & \left(s[I]+[M]^{-1}[K]\right)^{-1}[M]^{-1} \\
& \times\left(\left\{N_{0}\right\}^{T}+\frac{1}{s}\{P\}^{T}\right) .
\end{aligned}
$$

If we diagonalize the resultant matrix of the product $[M]^{-1}[K]$, it is possible to substitute it by the similarity transformation $[M]^{-1}[K]=[O]\left[D\left(\lambda_{i}\right)\right][O]^{-1}$, where $\left[D\left(\lambda_{i}\right)\right]=\operatorname{diag}\left(\lambda_{1}, \ldots, \lambda_{L}\right)$ represents a diagonal matrix, whose diagonal elements are, in this case, the eigenvalues of $[M]^{-1}[K]$, and $[O]$ is the corresponding eigenvectors' matrix. Taking into account this transformation, (7) comes into the following expression:

$$
\begin{aligned}
\{\hat{N}(s)\}^{T}=[O]\left[D\left(\frac{1}{s+\lambda_{i}}\right)\right][O]^{-1} & \\
& \times[M]^{-1}\left(\left\{N_{0}\right\}^{T}+\frac{1}{s}\{P\}^{T}\right) .
\end{aligned}
$$

As a final step, we apply the inverse Laplace transform to (8) in order to obtain the corresponding equation in time domain

$$
\begin{aligned}
\{n(t)\}^{T} & =[O]\left[D\left(e^{-\lambda_{i} t}\right)\right][O]^{-1}[M]^{-1}\left\{N_{0}\right\}^{T} \\
& +[O]\left[D\left(\frac{1}{\lambda_{i}}\left(1-e^{-\lambda_{i} t}\right)\right)\right][O]^{-1}[M]^{-1}\{P\}^{T}
\end{aligned}
$$

where vector $\{n(t)\}$ keeps the time-dependent coefficients which belong to the representation of the electron density as a linear combination of basis functions $\{T(\mathbf{r})\}$

$$
n(\mathbf{r}, t)=\sum_{i}\{n(t)\}_{i}\{T(\mathbf{r})\}_{i} .
$$

This way, through (10), we obtain the space-time distribution of the electron density.

Equations (9) and (10) show that the temporal evolution of the electron density is governed entirely by the eigenvalues of the matrix $[M]^{-1}[K]$. For a certain power, the existence of at least one negative eigenvalue of $[M]^{-1}[K]$ determines corona breakdown, since the electron density will grow exponentially in time. Thus, we can define a univocal breakdown criterion based on the eigenvalues of the matrix $[M]^{-1}[K]$, whose diagonalization is sufficient to determine if the discharge takes place. In order to determine the breakdown threshold, an iterative procedure has been followed: if, for a particular $\mathrm{E}$ field a negative eigenvalue exists, the E field is decreased, otherwise, it is increased.

\section{RESULTS}

As a first validation example, we have compared our algorithm to the theoretical results presented in [7] for an infinite circular waveguide. As shown in Fig. 1, the agreement between 


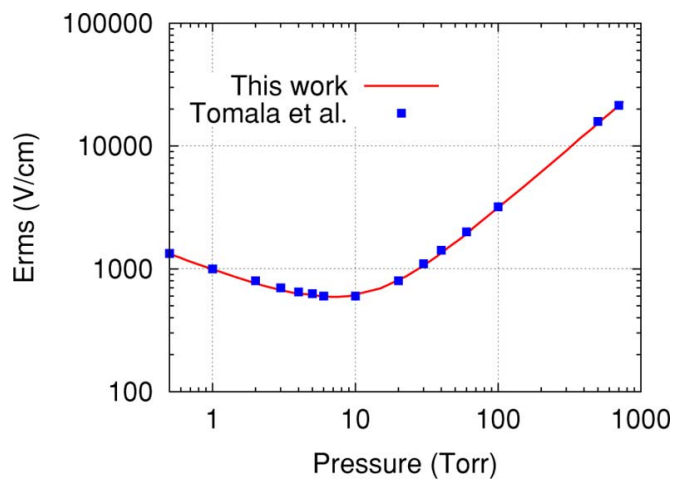

Fig. 1. Gas breakdown electric field onset in a circular waveguide with radius equal to $1 \mathrm{~cm}$. The frequency is set to $10 \mathrm{GHz}$ and the environment gas is dry air.

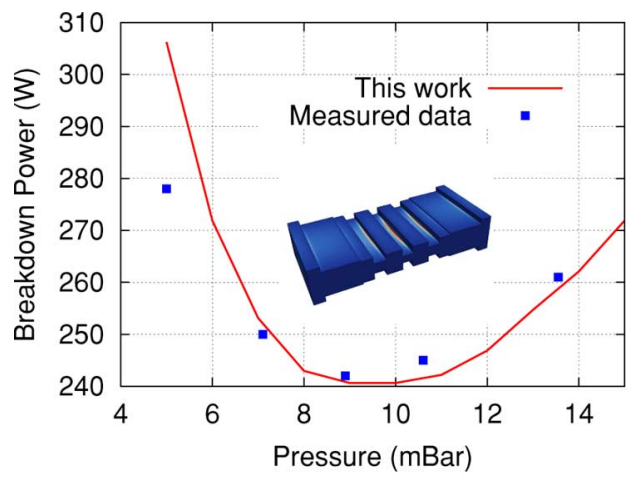

(a)

Fig. 2. Breakdown power threshold versus pressure at $9.5 \mathrm{GHz}$ for air.

both theoretical/numerical results is excellent, thus proving that our algorithm has been successfully applied.

As a second example, we have considered a lowpass filter based on rectangular waveguide for which the 3D structure and the calculated breakdown RF power at $9.5 \mathrm{GHz}$ are shown in Fig. 2. It is seen that the agreement between experimental (extracted from [8]) and numerical results is very good. In this case, only validation data around the minimum of the Paschen curve were available for comparison.

Once the numerical approach has been experimentally validated, a more complex structure has been studied. In particular, a 10-tooth waffle-iron filter extracted from [9] and shown in Fig. 3(a) has been considered. The S-parameter response and the electric field distribution for an input power of $1 \mathrm{~W}$ are respectively shown in Fig. 3(a) and (b). Considering that the filter is filled with air, we have calculated the breakdown power threshold for $6 \mathrm{GHz}$, which is represented in Fig. 3(b).

\section{CONCLUSION}

In this letter we have derived an algorithm for the prediction of the gas discharge breakdown threshold inside microwave components, e.g., filters, with arbitrary geometries. Theoretical results and measurements have been provided for several test structures, showing very good agreement. A special case of a

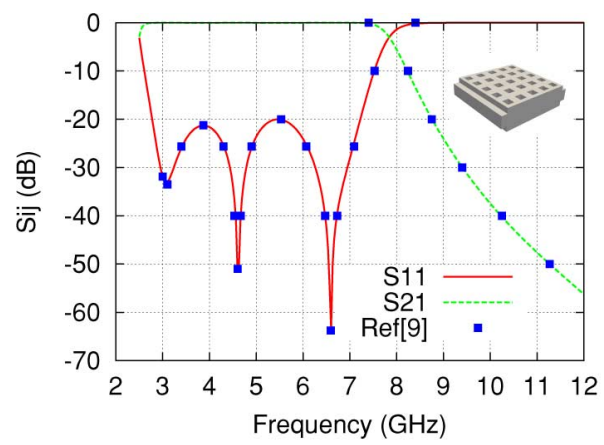

(a)

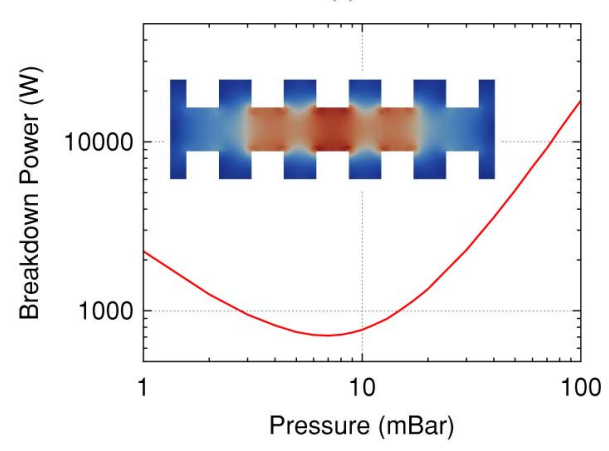

(b)

Fig. 3. (a) Waffle-iron filter with S-parameter response compared to the results from [9]. (b) Breakdown power threshold versus pressure at $6 \mathrm{GHz}$ for air together with the transversal cross section of the electric field magnitude (at the center of the filter in the propagation direction).

waffle-iron filter has been finally presented in order to show the flexibility of the numerical method to cope with very complex waveguide structures.

\section{REFERENCES}

[1] A. D. MacDonald, Microwave Breakdown in Gases. New York: Wiley, 1966.

[2] T. Olsson, D. Anderson, U. Jordan, and M. Lisak, "Microwave breakdown in air-filled resonators," in IEEE MTT-S Int. Dig., 1999, pp. 915-918.

[3] C. Vicente, M. Mattes, D. Wolk, B. Mottet, H. L. Hartnagel, J. Mosig, and D. Raboso, "Prediction of corona discharge: Towards a simulation tool for arbitrary geometries," in Proc. 13th Int. Symp. Antennas, Nice, France, Nov. 2004, pp. 326-327.

[4] G. Conciauro, M. Guglielmi, and R. Sorrentino, Advanced Modal Analysis-CAD Techniques for Waveguide Components and Filters. New York: Wiley, 2000.

[5] S. Cogollos, S. Marini, V. E. Boria, P. Soto, A. Vidal, A. Esteban, J. V. Morro, and B. Gimeno, "Efficient modal analysis of arbitrarily shaped waveguides composed of linear, circular, and elliptical arcs using the BIRME method," IEEE Trans. Microw. Theory Tech., vol. 51, no. 12, pp. 2378-2390, Dec. 2003.

[6] W. Woo and J. DeGroot, "Microwave absorption and plasma heating due to microwave breakdown in the atmosphere," IEEE Phys. Fluids, vol. PF-27, no. 2, pp. 475-487, 1984.

[7] R. Tomala, U. Jordan, D. Anderson, and M. Lisak, "Microwave breakdown of the TE11 mode in a circular waveguide," J. Phys. D: Appl. Phys., vol. 38, pp. 2378-2381, Jul. 2005.

[8] "Multipactor and Corona Discharge: Simulation and Design in Microwave Components," Final Rep., ESA Contract 16827/02/NL/EC, 2006.

[9] M. B. Manuilov and K. V. Kobrin, "Field theory CAD of waffle-iron filters," in Proc. 35th Eur. Microw. Conf., Oct. 2005, vol. 2, pp. $1227-1230$. 